\title{
ADAPTIVE ROBUST BACKSTEPPING NONLINEAR ALGORITHM APPLIED TO SHIP STEERING
}

\author{
Jialu Du, Chen Guo and Chengen Yang \\ School of Automation and Electrical Engineering, Dalian Maritime University, \\ Dalian 116026, P.R. China, E-mail: dujl@newmail.dlmu.edu.cn, \\ guoc@dlmu.edu.cn, chengeny@dlmu.edu.cn
}

\begin{abstract}
An adaptive robust nonlinear steering controller for ship's course is developed. Incorporating the Nussbaum-type function gain and adaptive backstepping algorithm, the proposed control strategy requires neither known dynamic parameters of a ship's model nor any knowledge about environmental disturbances. It is proved that the designed adaptive robust controller of ship steering can guarantee that the uniform ultimate boundedness of the resulting closed-loop system signals. Dynamic performance can be improved by an appropriate choice of the design parameters. The effectiveness of the presented algorithm has been demonstrated in a simulation involving a ship of $45 \mathrm{~m}$ in length. Copyright $(2005$ IFAC
\end{abstract}

Keywords: ship control, adaptive, robust, nonlinear, tracking.

\section{INTRODUCTION}

The ship course control directly influences the manoeuvrability, economy and security of ship navigation. An autopilot is a ship's steering controller, which automatically manipulates the rudder to decrease the errors between the desired heading angle and the actual heading angle. Conventional autopilots are based on simple PID control. Modern autopilots using the LQG and $H_{\infty}$ control design techniques have been reported in the literature by a large number of authors (Fossen, 2000). In addition, a large number of other advanced control methods, such as model reference adaptive control (Amerongen, 1984) and neural network adaptive control (Unar and Murray-smith, 1999), have been trialled in ship steering applications since the 1980s. So far, the linear Nomoto model has been widely accepted in the design of a ship course controller. However, in some cases such as a coursechanging manoeuvre calling for large rudder movements, ship steering equations of motion are highly nonlinear. For a certain nonlinear ship model, a state feedback linearization control law can be designed (Fossen, 1993), while feedback linearization with saturating and slew rate limiting actuators has been discussed by Tzeng et al. (1999). However they do not possess the robustness to changes of parameter and model. In order to maintain the desired performance of autopilots, it is important for designers to pay attention to the effects on a ship's motions of strong disturbances induced by wind, waves and currents, and to the robustness to changes in a ship's dynamics due to cargo loads, fouling and so on. A nonlinear adaptive autopilot based on backstepping and Nussbaum gain is developed for parametric uncertain ship without a priori knowledge of control gain (Du, 2004). A robust adaptive nonlinear control algorithm for ship steering autopilots, based on the projection approach and the Lyapunov stability theory, was presented by Yang (2000). However, the design procedure requires a priori knowledge of the signs of unknown control coefficients.

In this paper, an adaptive robust nonlinear control 
strategy for ship steering is presented based on the Nussbaum-type function (Nussbaum, 1983) and adaptive backstepping algorithm (Krstic, et al.,1995). An adaptive robust nonlinear controller of ship steering model with both completely unknown parameters and unknown disturbances. Uniform boundedness of all signals in the resulting closedloop course error system is guaranteed, and control law is smooth. Suitable transient performance and control action can be achieved by carefully adjusting the design parameters.

The paper is organized as follows: Section 2 discusses nonlinear models for ship steering with uncertainties of parameters and disturbances. In Section 3 the adaptive robust nonlinear controller of ship course-changing is presented. Section 4 contains the simulation study while Section 5 summarizes the conclusion.

\section{SHIP STEERING EQUATIONS OF MOTION}

For small rudder angles, the transfer function between the rudder angle $\delta$ and the yaw rate $r$ of a ship can be described by the linear 1 st order model of Nomoto et al. .

$$
\frac{r(s)}{\delta(s)}=\frac{K}{T s+1}
$$

$K$ is the gain constant and $T$ is time constant. The yaw angle $\psi(t)$ is related to the yaw rate $r(t)$ as: $\dot{\psi}(t)=r(t)$.

To describe large rudder angles and course-unstable ships, an extension of the 1st order model of Nomoto can be made by defining

$$
T \ddot{\psi}+\dot{\psi}+\alpha \dot{\psi}^{3}=K \delta
$$

where $\alpha$ is the Norrbin coefficient which is produced by reverse spiral manoeuvres (Fossen, 1994).

The ship's parameters are basically determined by its size and shape, and may vary depending on operational conditions such as ship speed, draft, trim, and water depth. The main environmental disturbances are wind, waves and currents, which are variable. Accordingly, the following nonlinear ship model is suggested in the controller design discussed in this paper.

$$
T \ddot{\psi}+\dot{\psi}+\alpha \dot{\psi}^{3}=K\left(\delta+\delta_{\omega}\right)
$$

where parameters $K, T, \alpha$ are unknown, $\delta_{\omega}$ represents an unknown bounded equivalent rudder angle induced by environmental disturbances such as waves, wind, ocean currents. $\delta_{\omega}$ is represented as

$$
\left|\delta_{\omega}\right| \leq p^{*}
$$

where $p^{*}$ is an unknown positive constant.

To meet the demands of the later design stages, state variables are selected as $x_{1}=\psi, x_{2}=\dot{\psi}$, and control variable as $u=\delta, y=\psi$ is output variable of the ship steering system. Then equation (3) is transformed into the following dynamic equations:

$$
\begin{gathered}
\dot{x}_{1}=x_{2} \\
\dot{x}_{2}=g u+W^{* T} \varphi\left(x_{2}\right)+g \delta_{\omega} \\
y=x_{1}
\end{gathered}
$$

where, $\quad g=K / T, W^{*}=[-1 / T,-\alpha / T]^{T}$, $\varphi\left(x_{2}\right)=\left[x_{2}, x_{2}^{3}\right]^{T}$. In equation (3), $T>0$ when a ship's line-movement is stable; whereas $T<0$ when a ship's line-movement is unstable. The sign of control coefficient $g$ is viewed as the unknown in this paper.

\section{DESIGN OF NONLINEAR ADAPTIVE ROBUST AUTOPILOT}

Ship autopilots can be designed to perform two entirely different functions: course-changing and course-keeping. Particular consideration is given to the course-changing problem in this paper. During course-changing operations it is desirable to specify the dynamics of the desired heading instead of using a constant reference signal. One simple way to do this is by applying model reference techniques. The ideal performance can be given by reference model (8) (Fossen, 1994)

$$
\psi_{d}=\frac{\omega_{n}^{2}}{s^{2}+2 \xi \omega_{n} s+\omega_{n}^{2}} \psi_{r}
$$

where $\xi$ and $\omega_{n}$ are the parameters to be determined that describe the closed-loop system response characteristics; the input $\psi_{r}$ of the reference model is the course-changing heading command, and the output $\psi_{d}$ of the reference model is the desired smooth course-changing signal. A second-order model is sufficient to generate the desired smooth course signal $\psi_{d}$ with known and bounded derivatives $\dot{\psi}_{d}, \ddot{\psi}_{d}$.

The adaptive robust nonlinear controller of course tracking of the system (5)-(7) is designed as follows. The design consists of 2 steps.

Step 1: Define the course heading error $z_{1}=x_{1}-\psi_{d}$, which satisfies 


$$
\dot{z}_{1}=x_{2}-\dot{\psi}_{d}
$$

$x_{2}$ is viewed as the virtual control input. The intermediate control function $\phi_{1}$ for the virtual control $x_{2}$ to stabilize (9) is defined as

$$
\phi_{1}\left(z_{1}\right)=-c_{1} z_{1}+\dot{\psi}_{d}
$$

where $c_{1}$ is a positive design constant. However, $x_{2}$ is not the actual control, hence a difference between $x_{2}$ and the intermediate control function $\phi_{1}$, which is defined as $z_{2}=x_{2}-\phi_{1}$. Accordingly, (9) should be expressed as:

$$
\dot{z}_{1}=-c_{1} z_{1}+z_{2}
$$

Consider Lyapunov function $V_{1}$

$$
V_{1}=\frac{1}{2} z_{1}^{2}
$$

The time derivative of $V_{1}$ along the solution of (11) is given by

$$
\dot{V}_{1}=-c_{1} z_{1}^{2}+z_{1} z_{2}
$$

The undesired effects of $z_{2}$ on $\dot{V}_{1}$ will be controlled at the next step.

Step 2: Using (9), (10) and the definition for $z_{2}$, the derivative of $z_{2}$ is expressed as

$$
\begin{aligned}
\dot{z}_{2}= & g u+W^{* T} \varphi\left(x_{2}\right)+g \delta_{\omega} \\
& +c_{1} x_{2}-c_{1} \dot{\psi}_{d}-\ddot{\psi}_{d}
\end{aligned}
$$

In the light of (4) and (14), the time derivative of $\frac{1}{2} z_{2}^{2}$ is

$$
\begin{aligned}
z_{2} \dot{z}_{2} \leq & z_{2}\left[g u+W^{* T} \varphi\left(x_{2}\right)+c_{1} x_{2}\right. \\
& \left.-c_{1} \dot{\psi}_{d}-\ddot{\psi}_{d}\right]+\left|z_{2}\right| g p^{*}
\end{aligned}
$$

For notional convenience, let $W_{a}^{*}=\left[\begin{array}{ll}1 & W^{* T}\end{array}\right]^{T}$,

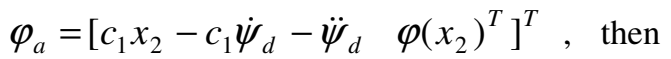
can be further written as

$$
z_{2} \dot{z}_{2} \leq z_{2}\left[g u+W_{a}^{* T} \varphi_{a}\left(x_{2}\right)\right]+\left|z_{2}\right| b^{*}
$$

where $b^{*}=g p^{*}$.

In order to cope with the unknown sign of control coefficient $g$, the Nussbaum gain technique is employed in this paper. A differentiable function $N(k)$ is called a Nussbaum-type function if it has the following properties:

$$
\begin{aligned}
& \limsup _{s \rightarrow \infty} \frac{1}{s} \int_{0}^{s} N(k) d k=\infty \\
& \liminf _{s \rightarrow \infty} \frac{1}{s} \int_{0}^{s} N(k) d k=-\infty
\end{aligned}
$$

Commonly used Nussbaum functions include: $\exp \left(k^{2}\right) \cos ((\pi / 2) k), k^{2} \cos (k), k^{2} \sin (k)$ (Ge and Wang, 2002).

To complete the later controller design, the following lemma relating to the property of Nussbaum gain and two useful technical lemmas are given.

Lemma1: (Ge and Wang, 2002) Let $V(\cdot)$ and $k(\cdot)$ be smooth functions defined on $\left[0, t_{f}\right)$ with $V(t) \geq 0, \forall t \in\left[0, t_{f}\right)$, and $N(\cdot)$ be an even smooth Nussbaum-type function. If the following inequality holds:

$$
\begin{array}{r}
V(t) \leq C_{0}+e^{-C_{1} t} \int_{0}^{t}(\theta N(k)+1) \dot{k} e^{C_{1} \tau} d \tau \\
\forall t \in\left[0, t_{f}\right)
\end{array}
$$

where constant $C_{1}>0, \theta$ is a nonzero constant and $C_{0}$ represents a suitable constant, then $V(t)$, $k(t)$ and $\int_{0}^{t} \theta N(k) \dot{k d} \tau$ must be bounded on $\left[0, t_{f}\right)$.

Consider Lyapunov function $V_{20}$

$$
\begin{aligned}
V_{20}= & \frac{1}{2} z_{2}^{2}+\frac{1}{2}\left(\hat{W}_{a}-W_{a}^{*}\right)^{T} \Gamma^{-1}\left(\hat{W}_{a}-W_{a}^{*}\right) \\
& +\frac{1}{2 \lambda}\left(\hat{b}-b^{*}\right)^{2}
\end{aligned}
$$

where $\Gamma=\Gamma^{T}>0, \lambda>0$, and $\hat{W}_{a}$ and $\hat{b}$ are the parameter estimates of $W_{a}^{*}$ and $b^{*}$ respectively to be determined later.

Let $u$ be

$$
\begin{gathered}
u=N(k)\left(c_{2} z_{2}+\hat{W}_{a}^{T} \varphi_{a}+\hat{b} \tanh \left(\frac{z_{2}}{\varepsilon}\right)\right) \\
N(k)=k^{2} \cos (k) \\
\dot{k}=c_{2} z_{2}^{2}+z_{2} \hat{W}_{a}^{T} \varphi_{a}+z_{2} \hat{b} \tanh \left(\frac{z_{2}}{\varepsilon}\right)
\end{gathered}
$$


where $c_{2}$ is a positive design constant, $\mathcal{E}$ is a small positive constant and $N(k)$ is an even smooth Nussbaum-type function.

In order to prevent parameter drifts, the following adaptive law incorporating a leakage term based on a variation of $\sigma$-modification is presented. Let the parameter adaptation laws be

$$
\begin{gathered}
\dot{\hat{W}}_{a}=\Gamma \varphi_{a} z_{2}-\Gamma \sigma_{w}\left(\hat{W}_{a}-W_{a}^{0}\right) \\
\dot{\hat{b}}=\lambda z_{2} \tanh \left(\frac{z_{2}}{\varepsilon}\right)-\lambda \sigma_{b}\left(\hat{b}-b^{0}\right)
\end{gathered}
$$

where $\sigma_{w}, \sigma_{b}, W_{a}^{0}, b^{0}>0$ are design constants.

Noting (16), (21), (23), the time derivative of $V_{20}$ is

$$
\begin{aligned}
\dot{V}_{20}= & z_{2} \dot{z}_{2}+\left(\hat{W}_{a}-W_{a}^{*}\right)^{T} \Gamma^{-1} \dot{\hat{W}}_{a}+\frac{1}{\lambda}\left(\hat{b}-b^{*}\right) \dot{\hat{b}} \\
& \leq g N(k) \dot{k}+z_{2} W_{a}^{*} \varphi_{a}+\left|z_{2}\right| b \\
& +\left(\hat{W}_{a}-W_{a}^{*}\right)^{T} \Gamma^{-1} \dot{\hat{W}}_{a}+\frac{1}{\lambda}\left(\hat{b}-b^{*}\right) \dot{\hat{b}}
\end{aligned}
$$

Adding and subtracting (27) on the right hand of (26), and using (23)-(25),

$$
c_{2} z_{2}^{2}+z_{2} \hat{W}_{a}^{T} \varphi_{a}+z_{2} \hat{b} \tanh \left(\frac{z_{2}}{\varepsilon}\right)
$$

there is

$$
\begin{aligned}
\dot{V}_{20} \leq & -c_{2} z_{2}^{2}+g N \dot{k}+\dot{k}+b^{*}\left|z_{2}\right|-\hat{b} z_{2} \tanh \left(\frac{z_{2}}{\varepsilon}\right) \\
& -z_{2}\left(\hat{W}_{a}-W_{a}^{*}\right)^{T} \varphi_{a}+\left(\hat{W}_{a}-W_{a}^{*}\right)^{T} \Gamma^{-1} \hat{\hat{W}}_{a} \\
& +\frac{1}{\lambda}\left(\hat{b}-b^{*}\right) \dot{\hat{b}} \\
\leq & -c_{2} z_{2}^{2}+g N \dot{k}+\dot{k}+b^{*}\left|z_{2}\right|-b^{*} z_{2} \tanh \left(\frac{z_{2}}{\varepsilon}\right) \\
- & \left(\hat{W}_{a}-W_{a}^{*}\right)^{T} \sigma_{w}\left(\hat{W}_{a}-W_{a}^{0}\right) \\
& -\left(\hat{b}-b^{*}\right) \sigma_{b}\left(\hat{b}-b^{0}\right)
\end{aligned}
$$

The following inequalities can be achieved by applying Young's inequality.

$$
\begin{aligned}
& \left(\hat{W}_{a}-W_{a}^{*}\right)^{T}\left(\hat{W}_{a}-W_{a}^{0}\right) \\
\geq & \frac{1}{2}\left\|\hat{W}_{a}-W_{a}^{*}\right\|^{2}-\frac{1}{2}\left\|W_{a}^{*}-W_{a}^{0}\right\|^{2} \\
& \left(\hat{b}-b^{*}\right)\left(\hat{b}-b^{0}\right) \\
\geq & \frac{1}{2}\left(\hat{b}-b^{*}\right)^{2}-\frac{1}{2}\left(b^{*}-b^{0}\right)^{2}
\end{aligned}
$$

By using (29), (30) and the property of function $\tanh (\cdot): 0 \leq|x|-x \tanh \left(\frac{x}{\varepsilon}\right) \leq 0.2785 \varepsilon$ for any $\varepsilon>0$ and $x \in R$, equation (28) can be further written as:

$$
\begin{aligned}
\dot{V}_{20} \leq & -c_{2} z_{2}^{2}+g N \dot{k}+\dot{k}+b^{*} 0.2785 \varepsilon \\
& -\frac{1}{2} \sigma_{w}\left\|\hat{W}_{a}-W_{a}^{*}\right\|^{2}-\frac{1}{2} \sigma_{w}\left\|\hat{W}_{a}-W_{a}^{*}\right\|^{2} \\
& -\frac{1}{2} \sigma_{b}\left(\hat{b}-b^{*}\right)^{2}+\frac{1}{2} \sigma_{b}\left(b^{*}-b^{0}\right)^{2} \\
\leq & -c_{2} z_{2}^{2}+g N \dot{k}+\dot{k}-\frac{1}{2} \sigma_{w}\left\|\hat{W}_{a}-W_{a}^{*}\right\|^{2} \\
& -\frac{1}{2} \sigma_{b}\left(\hat{b}-b^{*}\right)^{2}+C_{21}
\end{aligned}
$$

where the constant $C_{21}>0$ is defined as

$$
\begin{aligned}
C_{21}= & b^{*} 0.2785 \varepsilon+\frac{1}{2} \sigma_{W}\left\|W_{a}^{*}-W_{a}^{0}\right\|^{2} \\
& +\frac{1}{2} \sigma_{b}\left(b^{*}-b^{0}\right)^{2}
\end{aligned}
$$

Consider the augmented Lyapunov function $V_{2}$

$$
V_{2}=V_{1}+V_{20}
$$

Then, the time derivative of $V_{2}$ is

$$
\begin{aligned}
\dot{V}_{2} \leq & -c_{1} z_{1}^{2}+z_{1} z_{2}-c_{2} z_{2}^{2}+g N \dot{k}+\dot{k} \\
& -\frac{1}{2} \sigma_{w}\left\|\hat{W}_{a}-W_{a}^{*}\right\|^{2}-\frac{1}{2} \sigma_{b}\left(\hat{b}-b^{*}\right)^{2}+C_{21} \\
\leq & -\left(c_{1}-\frac{1}{4 a^{2}}\right) z_{1}^{2}-\left(c_{2}-a^{2}\right) z_{2}^{2}+g N \dot{k}+\dot{k} \\
& -\frac{1}{2} \sigma_{w}\left\|\hat{W}_{a}-W_{a}^{*}\right\|^{2}-\frac{1}{2} \sigma_{b}\left(\hat{b}-b^{*}\right)^{2}+C_{21} \\
& \leq-C_{22} V_{2}+C_{21}+g N \dot{k}+\dot{k}
\end{aligned}
$$

where the constant $C_{22}>0$ is defined as

$$
\begin{array}{r}
C_{22}=\min \left\{2\left(c_{1}-1 /\left(4 a^{2}\right)\right), 2\left(c_{2}-a^{2}\right),\right. \\
\left.\frac{\sigma_{w}}{\lambda_{\max }\left(\Gamma^{-1}\right)}, \sigma_{b} \lambda\right\}, c_{1} c_{2}>\frac{1}{4}, \forall a
\end{array}
$$

Upon multiplication of (34) by $e^{C_{22} t}$, it becomes

$$
\frac{d}{d t}\left(V_{2} e^{C_{22} t}\right) \leq\left(C_{21}+(g N+1) \dot{k}\right) e^{C_{22} t}
$$

Let $\rho=C_{21} / C_{22}$, integrating (39) over $[0, t]$, then 


$$
\begin{aligned}
0 \leq V_{2}(t) \leq & e^{-C_{22} t} V_{2}(0)+\rho\left(1-e^{-C_{22} t}\right) \\
& +e^{-C_{22} t} \int_{0}^{t}(g N+1) \dot{k} e^{C_{22} \tau} d \tau \\
\leq & V_{2}(0)+\rho \\
& +e^{-C_{22} t} \int_{0}^{t}(g N+1) \dot{k} e^{C_{22} \tau} d \tau
\end{aligned}
$$

Applying Lemma 1 to (39), it can be concluded that $\int_{0}^{t} g N \dot{k} d \tau, k(t)$ and $V_{2}(t)$ are bounded, hence $\hat{W}_{a}, \hat{b}, z_{1}, z_{2}, \phi_{1}$ and the original states $x_{1}, x_{2}$ are bounded $\left[0, t_{f}\right)$. According to (Ryan, 1991, Prop. 2 ), if the solution of the closed-loop is bounded, then $t_{f}=\infty$. All signals of the resulting closed-loop system are uniformly ultimately bounded. Furthermore, from the definition of $V_{2}(t)$ and (37), it is concluded that for any given $\mu^{*} \geq \sqrt{2\left(V_{2}(0)+\rho+C\right)}$, where $C$ is the upper bound of $e^{-C_{22} t} \int_{0}^{t}(g N+1) \dot{k} e^{C_{22} \tau} d \tau$, there exists $T$ such that, for all $t \geq T$, there is $\|z\| \leq \mu^{*}$. The compact set $\Omega_{z}=\left\{z \in R^{2}:\|z\| \leq \mu^{*}\right\}$ can be made as small as required by an appropriate choice of the design constants for achieving suitable transient performance. Hence, the realm of heading error $z_{1}=y-\psi_{d}$ is regulated to a small neighbourhood of $z_{1}=0$, as shown by the following theorem about ship course control systems.

Theorem 1: Applied to ship motion nonlinear systems (5)-(7) with parametric uncertainty and unknown boundedness of environmental disturbances, and without a priori knowledge of control direction, the adaptive robust nonlinear control scheme described by (21)-(25) guarantees that all signals of the resulting closed-loop adaptive system are uniformly ultimately bounded, and the tracking error of a ship's course can be regulated to any prescribed accuracy by appropriately choosing the design constants.

\section{SIMULATION STUDY}

This section validates the proposed nonlinear adaptive robust autopilot on a ship with a length of 45m (Unar and Murray-smith, 1999). The model equation (3) of the ship at a forward speed of $5 \mathrm{~m} / \mathrm{s}$ has the following set of dynamic parameters: $T=31 \mathrm{~s}, \quad K=0.51 / \mathrm{s}, \quad \alpha=0.4 \mathrm{~s}^{2} . \delta_{\omega}$ is a $8^{\circ} \times \operatorname{rand}(\cdot)$ with $\operatorname{rand}(\cdot)$ being zero mean random noise with magnitudes of 1 and frequency of
$0.1 \mathrm{rad} / \mathrm{s}$ which is a rudder angle that is equivalent to disturbances induced by wind, wave, current. In the simulation, let the input $\psi_{r}$ of the reference model (8) be the square wave signal whose period is $400 \mathrm{~s}$ and magnitude is $30^{\circ}$. The design parameters of the reference model are chosen as $\omega_{n}=0.05 \mathrm{rad} / \mathrm{s}$, $\xi=0.8$. The control design parameters are selected as $c_{1}=0.05, c_{2}=5, \Gamma=\operatorname{diag}([0.5,0.5,0.5])$, $\lambda=0.5, \quad \sigma_{b}=0.1, \quad \sigma_{w}=0.1, \quad \varepsilon=0.1$, $b^{0}=0.1, W_{a}^{0}=\left[\begin{array}{lll}0.1 & 0.01 & 0\end{array}\right]^{T}$. The initial values are chosen as $x_{1}(0)=x_{2}(0)=0, \hat{b}(0)=0$, $\hat{W}_{a}(0)=\left[\begin{array}{lll}0 & 0 & 0\end{array}\right]^{T}, k(0)=0.6 * \pi \quad$. The results of simulation are plotted in Fig. 1 to Fig. 6. Fig. 1 shows the actual heading $\psi$ (solid line) quickly tracks the desired heading $\psi_{d}$ (dotted line), and the control rudder angle (dashdot-line) is smooth. The parameter estimates $\hat{b}$ and $\hat{W}_{a}$, Nussbaum gain $N(k)$ (solid line) and its argument $k$ (dotted line) are illustrated in Fig. 2 to Fig. 6., respectively. The parameter estimates are convergent. All signals are bounded as proven in Theorem 1. From the above fact, it might be concluded that the presented controller possesses some adaptability and robustness with respect to environment disturbances.

\section{CONCLUSION}

In order to improve the performance of ship autopilot, this paper firstly discusses a nonlinear mathematical model of a ship steering system with parametric uncertainties and unknown environmental disturbances. Secondly, a nonlinear adaptive robust course-changing autopilot is put forward. The main advantage of the proposed control scheme is that no a priori knowledge is required about the sign of the unknown control coefficient due to the incorporation of Nussbaum gain into the autopilot adaptive backstepping design. In addition, it can deal with the uncertainties of both parameters and boundedness of environmental disturbances. It has been proven that the proposed adaptive robust nonlinear control strategy can guarantee the uniform ultimate boundedness of the resulting closed-loop adaptive system signals and accomplish adaptive tracking control of ship course. The effectiveness of the proposed method was illustrated using a simulation study.

\section{ACKNOWLEDGMENTS}

This work was supported in part by the Ministry of Communications of P.R.C. under grant (200432922504), in part by the Specialized Research Fund for the Doctoral Program of Higher Education of P.R.C. under grant (20040151007). 


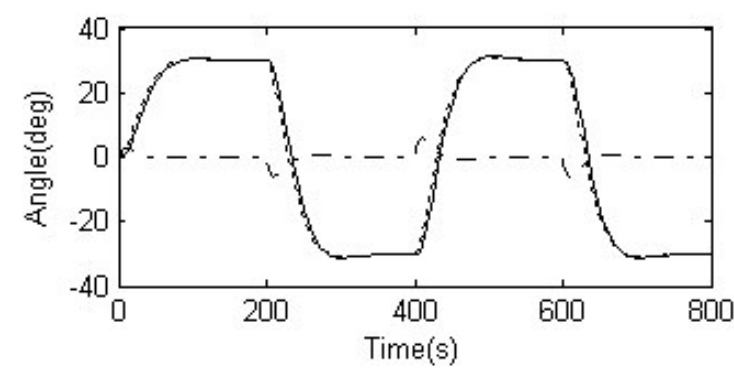

Fig. 1. Actual heading angle (-), reference heading angle $(\cdots)$, and control rudder angle $(-\cdot)$.

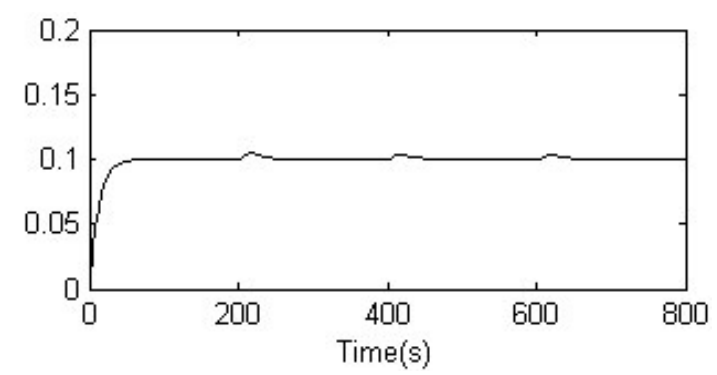

Fig. 2. Adapting parameter: $\hat{b}$.

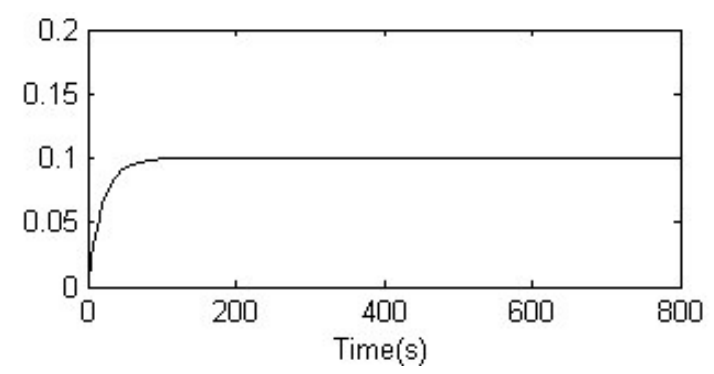

Fig. 3. Adapting parameter: $\hat{W}_{a, 1}$.

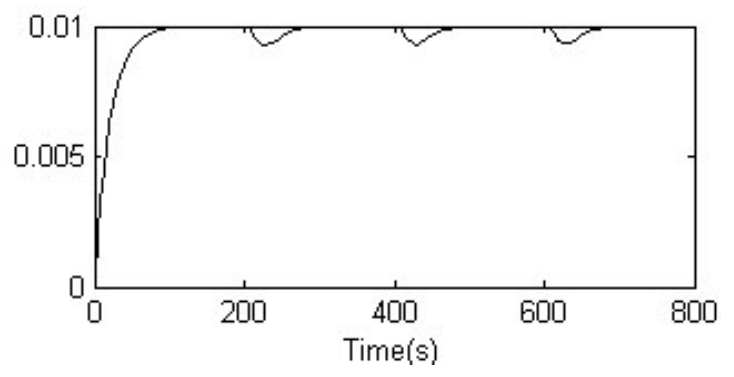

Fig. 4. Adapting parameter: $\hat{W}_{a, 2}$.

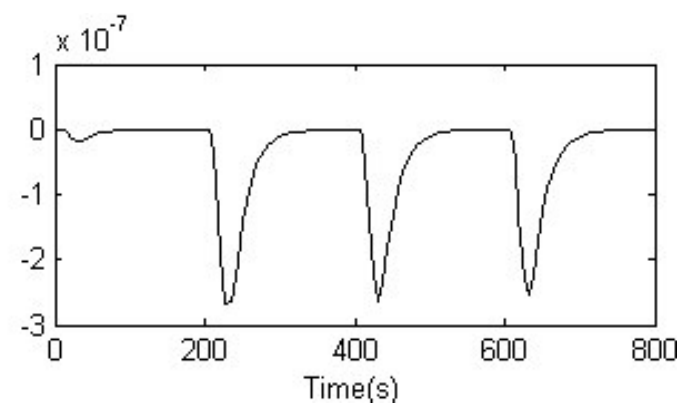

Fig. 5. Adapting parameter: $\hat{W}_{a, 3}$.

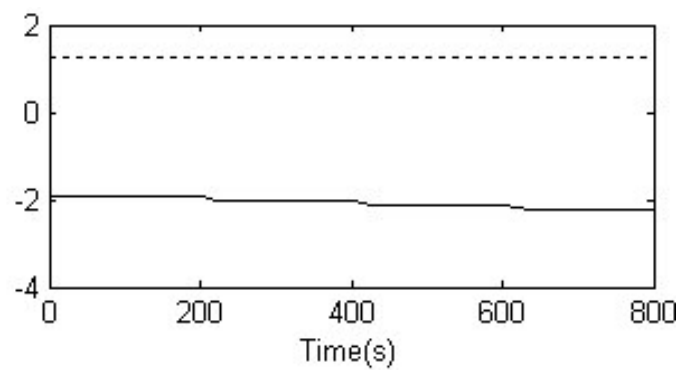

Fig. 6. Nussbaum gain $N(k)(-)$ and its argument $k(t)(\cdots)$.

\section{REFERENCES}

Amerongen J V. (1984). Adaptive steering of ships-a model reference approach. Automatica, 20(1), 314.

Du J. L., Guo C. (2004). Nonlinear adaptive ship course tracking control based on backstepping and Nussbaum gain. In Proceedings of the $23^{\text {th }}$ American Control Conference. Boston, Massachusetts. pp.3845-3850.

Fossen T. I. (1993). High performance ship autopilot with wave filter. In Proceedings of the 10th International Ship Control Systems Symposium. Ottawa, Canada. pp.2.271-2.285.

Fossen T. I. (1994). Guidance and control of ocean vehicles. John Wiley and Sons Ltd, New York.

Fossen T. I. (2000). A survey on nonlinear ship control: from theory to practice. In Proceedings of the 5th IFAC conference on manoeuvring and control of marine craft. Aalborg, Denmark. pp.116.

Ge S. Z., Wang J.(2002). Robust adaptive neural control for a class of perturbed strict feedback nonlinear systems. IEEE Trans. Neural Networks, 13(11), pp.1409-1419.

Krstic M, Kanellakopoulos I, Kokotovic P V (1995). Nonlinear and adaptive control design. Chap. 2. Wiley, New York.

Nussbaum R. D. (1983). Some remarks on the conjecture in parameter adaptive control. Syst. Contr. Lett., 3(3), 243-246.

Ryan E. P., (1991). A universal adaptive stabilizer for a class of nonlinear systems. Syst. Control Lett., 16(3), 209-218.

Tzeng C. Y., Goodwub G. C. and Crisafulli S. (1999). Feedback linearization of a ship steering autopilot with saturating and slew rate limiting actuator. Int. J. of Adaptive Control Signal Processing, 13(2), 23-30.

Unar M. A., Murray-smith D. J. (1999). Automatic steering of ships using neural networks. Int. J. of Adaptive Control Signal Processing, 13(2), 203218.

Yang Y. S. (2000). Robust adaptive control algorithm applied to ship steering autopilot with uncertain nonlinear system. Shipbuilding of China, 41(1), 21-25. 